\title{
THE CASE FOR ADVANCED DISTRIBUTED LEARNING
}

\section{Robert A. WISHER and J. Dexter FLETCHER}

$\mathrm{T}$ he U.S. Department of Defense launched the Advanced Distributed Learning (ADL) initiative in November 1997. It is the most recent and visible initiative in a long campaign to incorporate in practice the benefits of technology-based instruction and performance aiding - many of which benefits were created in the first place by defense related research and development. ADL is intended to accelerate large-scale development of dynamic and cost-effective learning environments and to stimulate a vigorous global market for these products. It is establishing a common technical framework for computer and Web-based learning that will foster the creation of reusable learning content as "instructional objects."

The goal of the ADL initiative is to ensure access to high quality education and training, tailored to individual needs, developed and delivered cost-effectively, available anytime and anywhere. This goal is viewed as something that can be achieved affordably, and thereby made feasible, only through the use of technology specifically computer technology. ADL is preparing for a world where communications networks and personal delivery devices are pervasive and inexpensive, as well as transparent to the users in terms of ease of use, bandwidth and portability. Much current ADL effort is an attempt to understand how best to utilize the next generation technology infrastructure for learning anytime, anywhere.

The drive to use technology to enhance a learning experience begins with an understanding of how people learn. Prominent reports from educational researchers argue that traditional instructor-centered approaches must be replaced with more active instruction involving leaner interactions. ${ }^{1}$ Researchers further specify the types of interactions that can occur in distributed learning environments. ${ }^{2}$ Examples include interactions to increase willingness to engage in learning, to increase participation, and to enhance elaboration and retention. A feature of effective interactions is that they must result in the transfer of knowledge or a change in intrinsic motivation. ADL 
can accommodate all of this interactivity in a manner that improves efficiency and reduces cost.

\section{The ADL Vision}

The ADL initiative is based on the view of future education, training, and performance aiding illustrated in Figure 1. As the figure suggests, this view, or 'vision', keys on three main components: (1) a global information infrastructure, with registered repositories populated by reusable instructional objects; (2) a server, which discovers, locates and then assembles instructional objects into education, training, and/or performance aiding materials tailored to user needs; and (3) devices that serve as personal learning associates on which the materials are presented.

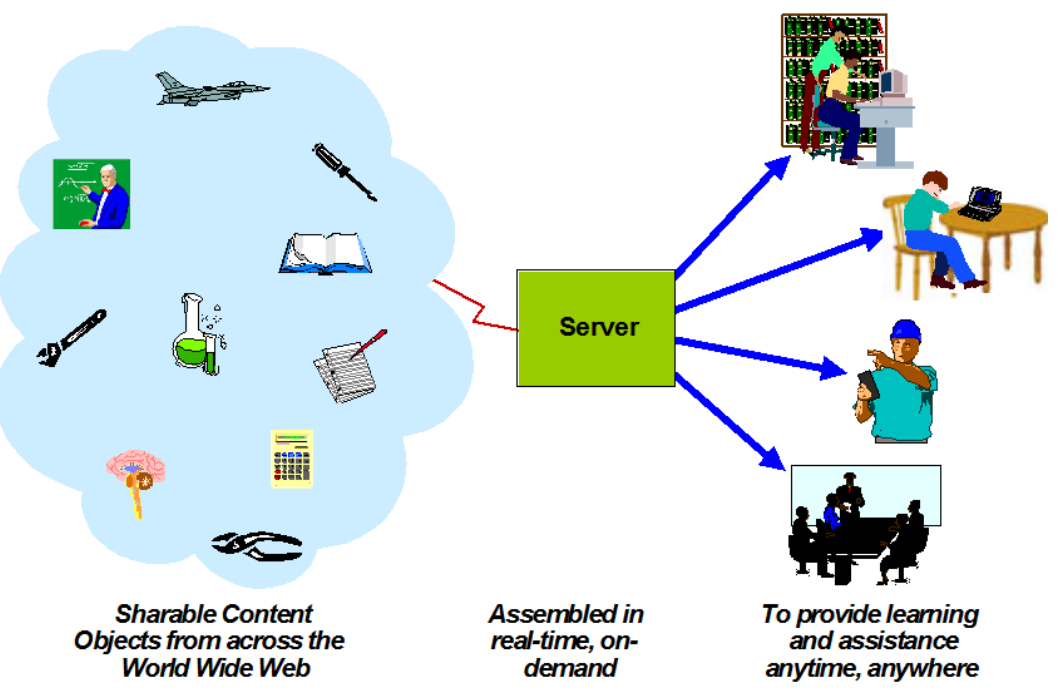

Figure 1: An Advanced Distributed Learning Future.

The server will assemble material on demand and in real time. This material will be tailored to the needs, capabilities, intentions, and learning state of each individual or group of individuals. Today, much of the work of the server is expected to be accomplished by 'middleware' in the form of learning management systems (LMSs). Within ADL, the term LMS implies a server-based environment in which the intelligence resides for controlling the delivery of learning content to students. The LMS knows what to deliver, when, and tracks student progress through the learning content. 


\section{Sharable Content Objects and Learning Management Systems}

To date most ADL effort has been devoted to the specification of reusable, sharable instructional objects. ${ }^{3}$ ADL development envisions the creation of learning libraries or repositories where learning objects may be accumulated and cataloged for broad distribution and use. Such repositories will provide the basis for a new instructional object economy that rewards content creators for developing high quality learning objects and assembling them into accessible, sharable, and adaptive learning experiences.

The development of reusable, sharable learning objects, then, is essential in achieving the ADL long-term vision. Among other things content must be separated from context-specific run-time constraints and proprietary systems so that it can be incorporated into other applications. Content must also have common interfaces and data. Content objects must, therefore, be:

- Durable: They should not require modification as versions of system software change;

- Interoperable: They should operate across a wide variety of hardware, operating systems and Web browsers;

- Accessible: It should be possible to index and find them as needed; and

- Reusable: It should be possible for many different development tools to modify and use them.

The key function of an LMS in the ADL context, then, is to manage content objects so that under the ADL initiative it should be possible for: A Web-based LMS to launch content that is authored by using tools from different vendors and to exchange data with that content, and for multiple Web-based LMS products/environments to access a common repository of executable content and to launch such content. The first function of launching content has largely been solved by ADL with the Sharable Content Object Reference Model, described later in this article.

In terms of common repositories of content, ADL is examining the Handle System ${ }^{4}$ as a comprehensive system for assigning, managing, and resolving persistent identifiers, known as "handles," for digital objects (i.e., instructional objects) and other resources on the Internet. The Handle System consists of a unique and persistent identifier for a resource and its owning organization, a protocol for resolving the location of the resource, and a reference implementation of the protocol so that a resource can always be found. The Handle System has the backing of the International Digital Object Identifier (DOI) Foundation, which provides a framework for managing intellectual content, such as electronic journal articles, images, and instructional objects. ${ }^{5}$ 


\section{The Sharable Content Reference Model (SCORM)}

Specification of ADL instructional content objects is being accomplished through the development of the Sharable Content Object Reference Model (SCORM). SCORM constitutes an important first step toward liberating learning content objects from local implementations. It is intended to provide specifications that enable content objects to be easily shared across multiple learning delivery environments.

Procedures for developing such content objects are within the state-of-the-art, but they must be articulated, accepted, and widely used as guidelines by developers and their customers. These goals can only be achieved through collaborative development. Collaboration will also increase the number, quality and per unit value of content objects made available. Such collaboration requires agreement upon a common reference model. The SCORM is intended to be such a model.

The SCORM assumes a Web-based infrastructure as a basis for its technical implementation. ADL made this assumption for several reasons. First, Web-based technologies and infrastructure are rapidly expanding and provide a mainstream basis for learning technologies; second, Web-based learning technology standards are not yet widespread; and third, Web-based content can be delivered using nearly any medium (e.g., CD-ROM, stand-alone systems). In other words, if it runs on the Web, it will run almost anywhere.

This approach embraces industry's transition to common content and delivery formats. The SCORM extends this trend to learning technologies. A successful sharable content object reference model must then support full articulation of guidelines that can be understood and implemented in the production of sharable content objects. It must also be adopted, understood, and used by as wide a variety of stakeholders as possible (courseware developers, courseware tool developers, and courseware customers, for example), and it must permit mapping of any stakeholder's model for instructional systems design and development into itself.

Much of the work required to create the SCORM is being done in a collaborative manner involving industry, academia, and governmental agencies, and on a global scale. The primary function of the ADL initiative in this process is to organize, encourage, orchestrate, and document their development efforts - and to ensure that defense education and training requirements are reflected in their work.

\section{Co-Labs}

The ADL initiative has established 'Collaboration-Laboratories' or Co-Labs in the U.S. at facilities in Alexandria, Virginia, Orlando, Florida, and Madison, Wisconsin to help achieve its vision. Additionally, ADL Partnership Co-Labs have been initially 
established in the United Kingdom and Canada. Other countries have expressed an interest in partnering with ADL. The Co-Labs help develop and test SCORM specifications and, more generally, determine how learning technologies can be best designed to bring about specific, targeted instructional outcomes reliably within as wide a range of instructional settings as possible. The ADL Co-Laboratories developed the SCORM conformance test software, procedures and supporting documents. The test software may be downloaded from the ADL website. ${ }^{6}$ In addition, ADL has developed a testing certification process for organizations that wish to provide a testing service for their community of interest. ADLNet tracks developments concerning the certification process. Within the first year of operation, more than forty vendors have been formally certified as being SCORM conformant.

\section{The Case for Technology}

The case for ADL instruction and interactive ADL technology may be roughly summarized as the following:

(1) Tailoring instruction (education and training) to the needs of individual students is imperative for efficient learning, but such efficiency has been unaffordable because it requires one instructor for each student.

Research has shown that students tutored one-on-one score about 2 standard deviations (or 'sigmas') higher on end-of-course achievement tests than students taught in one-on-many classrooms. ${ }^{7}$ This finding means for example and roughly that, with instructional time held fairly constant, one-on-one tutoring raised the performance of mid-level 50th percentile students to that of 98th percentile students. These and similar empirical research findings suggest that differences between oneon-one tutoring and typical classroom instruction are not only likely, but very large.

(2) ADL instruction and technology can, in many cases, make this instructional imperative affordable and, thereby, feasible.

Under any appreciable student load, it is less expensive to provide instruction with technology than to hire a sufficient number of tutors.

(3) ADL instruction is more effective than current instructional approaches in many settings across many subject matters.

We are not yet at the two standard deviation level, but analyses of more than 200 assessments of computer-based instruction showed an improvement of 0.32 sigma (roughly raising achievement from the 50th percentile level to the 63th percentile) for students using technology. ${ }^{8}$ For instructional delivery through the World Wide Web, an initial inquiry based on 47 studies ${ }^{9}$ indicated an improvement of 0.24 sigma (from roughly the 50th percentile to the 60th percentile). Another 44 assessments of 
interactive multimedia instruction ${ }^{10}$ produced an improvement of 0.50 sigma (from roughly the 50th percentile level to the 69th percentile), and five assessments of recent intelligent tutoring systems that directly mimic one-on-one instruction produced an improvement of 1.05 sigma (from roughly the 50th percentile level to the 85 th percentile). ${ }^{11}$

(4) ADL instruction is generally less costly than current instructional approaches, especially when many students or expensive devices are involved.

Reductions in operating and support costs average about 63 percent. In a review of 40 studies, savings in the time needed to achieve given instructional objectives averaged about 30 percent. ${ }^{12}$ If employed fully, these time-to-train savings could reduce the costs of specialized skill training in the Department of Defense by nearly one-fourth.

(5) ADL instruction is often the most cost-effective alternative for distributing instruction and for sustaining and enhancing the capabilities and readiness of military personnel after they are assigned to duty stations.

Travel, temporary duty, and permanent change of station costs can all be decreased by bringing instruction to learners rather than bringing learners to the instruction. More significantly, the time savings commonly found in technology-based instruction, allow personnel to be released earlier for operational duties - reducing personnel costs and increasing operational value.

(6) ADL instruction will become increasingly affordable and instructionally effective with the development and use of standardized instructional objects.

Early results indicate savings of about 50 percent, but the work is much too early to understand the full potential.

Overall, a rule of "thirds" emerges from assessments of technology-based instruction. That is to say that use of these ADL technologies reduces the cost of instruction by about one-third, and it either reduces time of instruction by about one-third or it increases the amount of skills and knowledge acquired by about one-third.

It should be emphasized that ADL capabilities can be used either by individuals or groups of individuals working in collaboration. The capabilities can be used in residential classrooms, remote classrooms, or any remote (distributed) locationworkplace, home, or elsewhere - outside of classroom walls. Further, it is neither a goal nor an expectation that ADL instruction will replace all human instructors. They will continue to be needed. However, their roles and responsibilities remain perennial issues in the design and implementation of ADL instruction. Finding the right balance is important. 


\section{The Server and Intelligent Tutoring Systems}

As many trainers will note, the greatest technical challenge for the ADL initiative is in construction of the Server shown in the middle of Figure 1. Help is on the way. Beginning in the late 1960's, and in parallel with research into computer-based instruction (CBI), groups of researchers began to explore the greater potential of 'information structure-oriented' approaches to represent human cognition and learning. The use of these structures to represent how we learn, master skills, and define subject domains eventually led to the development of an approach we now call Intelligent Tutoring Systems (ITS). ${ }^{13}$

'Intelligent' in the context of intelligent tutoring systems refers to the specific functionalities that are the goals of ITS development. These functionalities are distinct from those found in more conventional approaches to computer-based instruction. They require ITS to:

- Generate instruction in real time and on demand as required by individual learners;

- Support mixed initiative dialogue, allowing free form discussion between the technology and the student or user.

This generative approach is also the goal of the Advanced Distributed Learning initiative, which is intended to combine the benefits of object oriented development and Web delivery with those of technology-based instruction to achieve its objectives.

The ADL initiative and the development of ITS, then, have a number of key goals in common:

- Both are generative in that they envision the development of presentations on demand, in real time;

- Both are intended to tailor content, sequence, level of difficulty, level of abstraction, style, etc. to users' intentions, backgrounds, and needs;

- Both have a stake in research intended to accomplish such individualization;

- Both can be used equally well to aid learning or decision making;

- Both are intended to accommodate mixed initiative dialogue in which either the technology or the user can initiate or respond to inquiries in natural language;

- Both will benefit greatly from a supply of sharable instructional objects readily available for the generation of instructional (or decision aiding) presentations. 


\section{Web Development and ADL}

The World Wide Web has essentially reset the development agenda for both CBI and ITS development. It has established an ever-improving communications and delivery platform for accessing knowledge. Much of the development work once needed to adapt to the latest technology platform has been eliminated. The Web has become the universal delivery platform.

The use of Internet and Web standards and infrastructures has freed learning system developers to focus on next-generation learning architectures. The emerging semantic Web, which along with its ontology will allow us to export any knowledge representation system onto the web and link it to any other, will only strengthen this link - substantially. Discussions are underway within many standards organizations regarding next generation Web-based learning architectures. These discussions are expected to eventually result in implementable specifications.

\section{The Way Ahead}

The ADL initiative is intended to take advantage of the rapid growth of electronic commerce and the World Wide Web, and apply it to the needs of the learning community and life-long learners. It will help provide the learning resources that the defense community needs to ensure the operational effectiveness of its forces. It will help provide similar resources to all federal agencies, which also depend on human performance and competence. Cooperative development among all sectorsgovernment, private industry, and academia - is needed and is being used to achieve the goals of the ADL initiative. For example, ADL is seeking ways to integrate with simulations, through the High Level Architecture standard (IEEE Standard 1516). $\mathrm{ADL}$ is also seeking ways to integrate with multiplayer online games and collaborative learning environments. ${ }^{14}$

Users will (eventually) communicate with a personal learning associate using natural language dialogue initiated either by the device or by its users. It will be portable, perhaps small enough to be carried in a shirt pocket, or it may be the shirt itself. At present PDAs, laptops, and other personal computing capabilities are sufficient for ADL needs.

\section{Notes:}

1 Thomas M. Duffy and David H. Jonassen, "New implications for instructional technology," Instructional Technology 31, 5 (1991): 7-12.

2 Ellen Wagner, "In support of a functional definition of interaction," in Teaching and learning at a distance: What it takes to effectively design, deliver and evaluate programs, ed. Thomes E. Cyrs (San Francisco: Jossey Bass, 1997), 19-26. 
Philip V.W. Dodds and Schawn E. Thropp, Sharable Content Object Reference Model, SCORM 2004 Overview (ADL Resource Center), <http://www.adlnet.org/index.cfm? fuseaction $=$ rcdetails\&libid=648 $>$ (9 February 2004).

Corporation for National Research Initiatives, The Handle System, <http://www.handle. net/introduction.html> (9 February 2004).

International Digital Object Identifier (DOI) Foundation, The Digital Object Identifier System, < www.doi.org> (3 May 2004). http://adlnet.org/

Benjamin S. Bloom, "The 2 sigma problem: The search for methods of group instruction as effective as one-to-one tutoring," Educational Researcher 13 (1984): 4-16.

John A. Kulik, "Meta-analytic studies of findings on computer-based instruction," in Technology assessment in education and training, ed. Eva L. Baker and Harold F. O’Neil (Hillsdale: Lawrence Erlbaum Associates, 1994), 9-33.

Robert A. Wisher and Tatana M. Olson, The effectiveness of Web-based training, Research Report 1802 (Alexandria, Virginia, USA: U.S. Army Research Institute for the Behavioral and Social Sciences, 2003).

J. Dexter Fletcher, Effectiveness and cost of interactive videodisc instruction in defense training and education, Paper P-2372 (Alexandria, VA: Institute for Defense Analyses, 1990).

11 Beverly P. Woolf and Wes Regian, "Knowledge-based training systems and the engineering of instruction," in Training and retraining: A handbook for business, industry, government, and the military, ed. Sigmund Tobias and J. Dexter Fletcher (New York: Macmillan Reference USA, 2000), 339-356.

Fletcher, Effectiveness and cost.

13 Martha C. Polson and J. Jeffrey Richardson, eds., Foundations of Intelligent Tutoring Systems (Hillsdale, NJ: Lawrence Erlbaum Associates, 1988).

14 Curtis J. Bonk and Robert A. Wisher, Applying collaborative and e-learning tools to military distance learning: A research framework, Technical Report 1107 (Alexandria, VA: U.S. Army Research Institute for the Social and Behavioral Sciences, 2000).

ROBERT WISHER is Director of the Advanced Distributed Learning initiative, Office of the Secretary of Defense, Washington, DC, USA. He leads a network of ADL Co-Laboratories engaged in ADL. He received a doctorate degree in Cognitive Psychology from the University of California, San Diego. Dr. Wisher has published widely on the topic of training technologies. He is currently on the Editorial Board of The American Journal of Distance Education. For correspondence: Dr. Robert A. Wisher, Office of the Secretary of Defense, DUSD(R), 4000 Defense Pentagon, Room 1C757, Washington, DC 20301-4000. E-mail: Robert.Wisher@osd.mil.

J. DEXTER FLETCHER is with the Institute for Defense Analyses, Alexandria, Virginia, USA. Dr. Fletcher is a renowned world researcher and analyst in the fields of learning sciences, educational technology, and military training. He received his doctorate degree from Stanford University. He has since worked in the defense laboratories, in research universities, and in his current position at a leading military research institution. He recently was Co-Editor of Training and retraining: A handbook for business, industry, government, and military, published by Macmillan Reference USA. 